\title{
Maximum nullity and zero forcing of circulant graphs
}

https://doi.org/10.1515/spma-2020-0106

Received April 23, 2020; accepted October 27, 2020

\begin{abstract}
The zero forcing number of a graph has been applied to communication complexity, electrical power grid monitoring, and some inverse eigenvalue problems. It is well-known that the zero forcing number of a graph provides a lower bound on the minimum rank of a graph. In this paper we bound and characterize the zero forcing number of various circulant graphs, including families of bipartite circulants, as well as all cubic circulants. We extend the definition of the Möbius ladder to a type of torus product to obtain bounds on the minimum rank and the maximum nullity on these products. We obtain equality for torus products by employing orthogonal Hankel matrices. In fact, in every circulant graph for which we have determined these numbers, the maximum nullity equals the zero forcing number. It is an open question whether this holds for all circulant graphs.
\end{abstract}

Keywords: zero forcing, minimum rank, maximum nullity, circulant graph, bipartite graph, graph product.

MSC: 05C50, 05C75, 05C76, 15A03

\section{Introduction}

Let $G$ be a simple finite graph with vertex set $V(G)$ and edge set $E(G)$. Suppose in the graph $G$ some vertices are filled and some are unfilled. The filling rule is as follows: if a vertex $v \in V(G)$ is filled and has exactly one unfilled neighbor, $w$, then vertex $v$ forces $w$ to be filled, and $v$ is referred to as a forcing vertex. Given $F \subseteq V(G)$, the final filling of $F$ is the set of filled vertices obtained by initially filling the vertices of $F$ and leaving every vertex in $V(G) \backslash F$ unfilled and applying the filling rule until no more vertices can be filled. The set $F$ is called a zero forcing set if the final filling of $F$ is $V(G)$. The terminology of zero forcing arose in the context of forcing entries of a null vector to be zero as first described in [2]. An example of a zero forcing set is given in Figure 1.

In various applications, it is of interest to find the cardinality of a smallest zero forcing set in $G$ (which always exists since $V(G)$ is a trivial zero forcing set). The zero forcing number of $G$, denoted $Z(G)$, is the minimum cardinality of a zero forcing set for a graph $G$. Determining $Z(G)$ is NP-hard [1] in general, but has been calculated for some well-known classes of graphs (see, for example, [2, 14, 16]). Variations of zero forcing have been useful in communication complexity, quantum mechanics, electrical network monitoring, and

\footnotetext{
Linh Duong: Department of Mathematics, University of St. Thomas, St. Paul, MN, 55105, USA, E-mail: linhduong0527@gmail.com

Brenda K. Kroschel: Department of Mathematics, University of St. Thomas, St. Paul, MN, 55105, USA, E-mail: bkkroschel@stthomas.edu

Michael Riddell: Department of Mathematics \& Statistics, McMaster University, Hamilton, ON, L8S 4L8, Canada, E-mail: riddelmj@mcmaster.ca, michaeljamesriddell@gmail.com

*Corresponding Author: Kevin N. Vander Meulen: Department of Mathematics, Redeemer University, Ancaster, ON, L9K 1J4, Canada, E-mail: kvanderm@redeemer.ca

Adam Van Tuyl: Department of Mathematics \& Statistics, McMaster University, Hamilton, ON, L8S 4L8, Canada,

E-mail: vantuyl@math.mcmaster.ca
} 

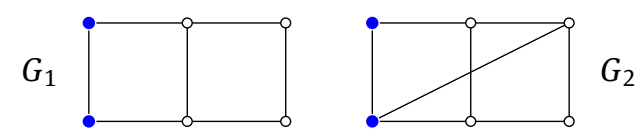

Figure 1: The filled vertices are a zero forcing set in $G_{1}$ but not in $G_{2}$.

some inverse eigenvalue problems (see $[5,14]$ for references). The zero forcing number originated in [2] as a technique to find a bound on the minimum rank of a symmetric matrix associated with a graph. Let $\mathcal{S}(G)$ denote the set of symmetric matrices over $\mathbb{R}$ whose graph is $G$. In particular, if $G$ is a graph with vertices $v_{0}, v_{1}, \ldots, v_{n-1}$, then $A \in \mathcal{S}(G)$ if $A$ is a real symmetric matrix such that for $i \neq j, A_{i j} \neq 0$ if and only if $v_{i}$ is adjacent to $v_{j}$ in $G$. Note that if $A \in \mathcal{S}(G)$, then there is no restriction on the diagonal entries of $A$. Let $M(G)=\max \{\operatorname{nullity}(A) \mid A \in \mathcal{S}(G)\}$. It was demonstrated in [2] that $Z(G)$ provides an upper bound on $M(G)$.

Theorem 1.1. [2] Let $G$ be a graph and let $F \subset V(G)$ be a zero forcing set of $G$. Then $M(G) \leq|F|$, and thus $M(G) \leq Z(G)$.

Recent work [3] describes families of graphs for which equality holds in Theorem 1.1, that is, families of graphs $G$ with $M(G)=Z(G)$. If we let $\operatorname{mr}(G)=\min \{\operatorname{rank}(A) \mid A \in \mathcal{S}(G)\}$, then the rank theorem tells us that $\operatorname{mr}(G)+$ $M(G)=n$. Hence Theorem 1.1 demonstrates that zero forcing can provide a lower bound on the minimum rank of any symmetric matrix associated with a graph.

Before going forward, we state some known facts about zero forcing, which can be found in [2].

\section{Lemma 1.2. [2]}

1. For any $k$-regular graph $G, Z(G) \geq k$.

2. For $n>1, M\left(K_{n}\right)=Z\left(K_{n}\right)=n-1$.

3. For $n \geq 3, Z\left(C_{n}\right)=M\left(C_{n}\right)=2$.

4. For $n \geq 5, Z\left(\overline{C_{n}}\right)=M\left(\overline{C_{n}}\right)=n-3$, where $\bar{G}$ denotes the complement of the graph $G$.

5. For disjoint graphs $G$ and $H, Z(G \cup H)=Z(G)+Z(H)$.

6. For the Cartesian product of graphs $G$ and $H, Z(G \square H) \leq \min \{|V(H)| Z(G),|V(G)| Z(H)\}$.

7. For $m \geq 2, M\left(K_{2} \square C_{m}\right)=Z\left(K_{2} \square C_{m}\right)=\min \{m, 4\}$.

In this paper we explore the zero forcing number for various classes of circulant graphs. Section 2 defines circulant graphs and reviews some of their properties. In addition, we extend Deaett and Meyer's results on consecutive circulants [10]. The maximum nullity and zero forcing number of circulant graphs that are bipartite is explored in Section 3. For every bipartite circulant $G$ considered in this section, $Z(G)=M(G)$. Section 4 introduces the torus product of a graph, noting that certain circulant graphs can be viewed as a torus product. The section explores the zero forcing number and maximum nullity for several cases of torus products. The Möbius ladder is a special case of a torus product. We note that for many circulant graphs $G$ which are torus products, the numbers $Z(G)$ and $M(G)$ are again equal, but there are still cases for which this is an open question. In Section 5, we show that all cubic circulant graphs $G, Z(G)=M(G)$, and we compute this value.

\section{Properties of circulant graphs}

We recall some of the properties of circulant graphs, and derive some basic results on the zero forcing number and minimum rank for this family. For standard graph theory terminology, see [21].

Given an integer $n \geq 1$ and a subset $S \subseteq\left\{1,2, \ldots,\left\lfloor\frac{n}{2}\right\rfloor\right\}$, a circulant graph $G=C_{n}(S)$ is a graph with vertex set $V(G)=\left\{v_{0}, v_{1}, \ldots, v_{n-1}\right\}$ and edge set $E(G)=\left\{\left\{v_{i}, v_{i+j}\right\} \mid i \in\{0, \ldots, n-1\}\right.$ and $\left.j \in S\right\}$, taking sub- 
scripts modulo $n$. Note that if $S=\left\{s_{1}, \ldots, s_{t}\right\}$, then we will abuse notation and write $C_{n}\left(s_{1}, \ldots, s_{t}\right)$ instead of $C_{n}\left(\left\{s_{1}, \ldots, s_{t}\right\}\right)$. Furthermore, we assume that $s_{1}<s_{2}<\cdots<s_{t}$. Some examples of circulant graphs are given in Figures 2 and 3.

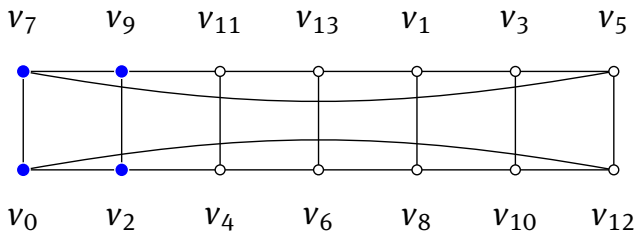

Figure 2: The graph $C_{14}(2,7) \cong K_{2} \square C_{7}$ (see Section 4) with a zero forcing set.
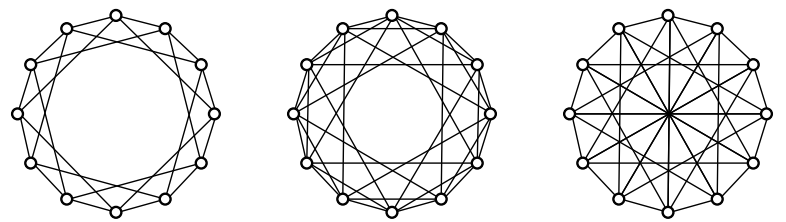

Figure 3: The circulant graphs $C_{12}(1,3), C_{12}(1,2,4)$ and $C_{12}(1,4,6)$.

Since circulant graphs are vertex transitive, they are regular graphs. In particular, if $G=C_{n}\left(s_{1}, \ldots, s_{t}\right)$, then $G$ is $(2 t-1)$-regular if $2 s_{t}=n$, and $2 t$-regular otherwise. Combining this observation with Lemma 1.2(1) gives a lower bound on the zero forcing number of a circulant graph:

Theorem 2.1. Suppose $G=C_{n}\left(s_{1}, \ldots, s_{t}\right)$. If $2 s_{t}=n$, then $Z(G) \geq 2 t-1$. If $2 s_{t} \neq n$, then $Z(G) \geq 2 t$.

Not every circulant graph is a connected graph (see Figure 4). The connected circulant graphs were characterized by Boesch and Tindell [6]; in the statement below, we write $g G$ to denote $g$ disjoint copies of the graph $G$.

Theorem 2.2. [6] If $G=C_{n}\left(s_{1}, s_{2}, \ldots, s_{t}\right)$, then $G$ is connected if and only if $\operatorname{gcd}\left(n, s_{1}, s_{2}, \ldots, s_{t}\right)=1$. If $\operatorname{gcd}\left(s_{1}, s_{2}, \ldots, s_{t}, n\right)=g$, then $C_{n}\left(s_{1}, s_{2}, \ldots, s_{t}\right) \cong g C_{\frac{n}{g}}\left(\frac{s_{1}}{g}, \frac{s_{2}}{g}, \ldots, \frac{s_{t}}{g}\right)$.

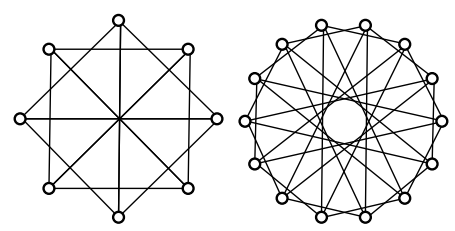

Figure 4: The disconnected circulant graphs $C_{8}(2,4) \cong 2 K_{4}=K_{4} \cup K_{4}$, and $C_{14}(2,6) \cong 2 C_{7}(1,3)$.

Using the function $f: \mathbb{Z}_{n} \rightarrow \mathbb{Z}_{n}$, defined by $f(x)=k x$, Muzychuk [19] proved the following graph isomorphism between circulant graphs. 
Lemma 2.3. [19] If $n>1$ and $\operatorname{gcd}(k, n)=1$, then $C_{n}\left(s_{1}, s_{2}, \ldots, s_{t}\right) \cong C_{n}\left(\left\{k s_{1}, k s_{2}, \ldots, k s_{t}\right\}\right){ }^{1}$

As an example of Lemma 2.3, if $n=7$ and $k=3$, then $C_{7}(1,3) \cong C_{7}(2,3)$. Likewise, taking $n=7$ and $k=5$ gives $C_{7}(1,3) \cong C_{7}(1,2)$.

As noted in the introduction, the zero forcing number (and minimum rank) of a number of families of graphs are known. Some of these families (e.g., complete graphs, cycles) are special cases of circulant graphs. The next theorem summarizes some of these known results.

Theorem 2.4. Let $G=C_{n}(S)$ be a circulant graph.

1. If $S=\{j\}$ and $\operatorname{gcd}(n, j)=1$, then $Z(G)=M(G)=2$.

2. If $S=\left\{1, \ldots,\left\lfloor\frac{n}{2}\right\rfloor\right\} \backslash\{j\}, \operatorname{gcd}(n, j)=1$, and $n \geq 5$, then $Z(G)=M(G)=n-3$.

3. If $S=\left\{1,2, \ldots,\left\lfloor\frac{n}{2}\right\rfloor\right\}$, then $Z(G)=M(G)=n-1$.

Proof. (1) The circulant graph $C_{n}(1) \cong C_{n}$, the $n$-cycle. By Lemma 2.3, $C_{n}(j) \cong C_{n}(1)$. The result then follows from Lemma 1.2(3).

(2) The graph $C_{n}\left(2, \ldots,\left\lfloor\frac{n}{2}\right\rfloor\right)$ is the complement of $C_{n}(1) \cong C_{n}$. If follows by Lemma 2.3, that if $S=$ $\left\{1, \ldots,\left\lfloor\frac{n}{2}\right\rfloor\right\} \backslash\{j\}$ and $\operatorname{gcd}(n, j)=1$, then $G \cong \overline{C_{n}}$. Now apply Lemma 1.2(4).

(3) Under this hypothesis, $G \cong K_{n}$; the conclusion follows from Lemma 1.2(2).

Given $n \geq 1$ and $1 \leq d \leq\left\lfloor\frac{n}{2}\right\rfloor$, the graphs $C_{n}(1,2, \ldots, d)$ are known as consecutive circulant graphs (e.g., see Figure 5). Deaett and Meyer [10] determined the zero forcing number and maximum nullity of consecutive circulants.

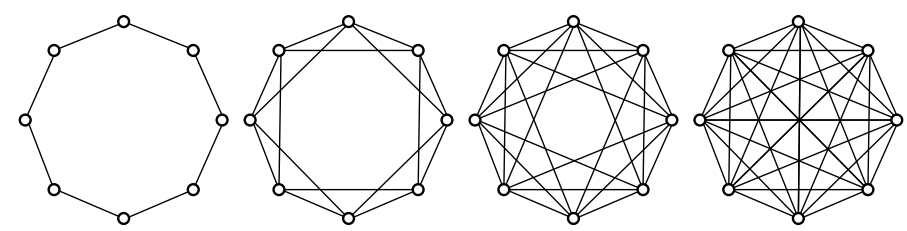

Figure 5: The consecutive circulants $C_{8}(1), C_{8}(1,2), C_{8}(1,2,3)$, and $C_{8}(1,2,3,4)$.

Theorem 2.5. [10, Theorems 5.4 and 5.7] If $G=C_{n}(1,2, \ldots, d), 1 \leq d \leq\left\lfloor\frac{n}{2}\right\rfloor$, then $M(G)=Z(G)=2 d$.

By combining the above result with Lemma 2.3, one can determine $Z(G)$ and $M(G)$ for some other families of circulant graphs:

Corollary 2.6. Suppose $G=C_{n}(s, 2 s, 3 s, \ldots, t s)$ for $1<t s<\frac{n}{2}$.

1. If $\operatorname{gcd}(n, s)=s$, then $M(G)=Z(G)=2$ st.

2. If $\operatorname{gcd}(n, s)=1$, then $M(G)=Z(G)=2 t$.

Proof. (1) If $s=1$, then the result holds by Theorem 2.5. Suppose $\operatorname{gcd}(n, s)=s \neq 1$. By Theorem 2.2, since $\operatorname{gcd}(n, s, 2 s, \ldots, t s) \neq 1$, the graph $G$ is disconnected. In particular, $G \cong s H$ with $H=C_{\frac{n}{s}}(1,2,3, \ldots, t)$. By Theorem 2.5 $M(H)=Z(H)=2 t$, so the result follows from Lemma 1.2(5).

(2) By Lemma $2.3, C_{n}(s, 2 s, \ldots, t s) \cong C_{n}(1,2, \ldots, t)$. Now apply Theorem 2.5. 


\section{Families of bipartite circulants}

In this section, we determine the zero forcing number and minimum rank of some families of bipartite circulant graphs using the work of Meyer [18]. Note that Meyer investigates the family of bipartite graphs whose biadjacency matrix is a circulant matrix. These graphs are sometimes called generalized bipartite circulants, although in [18], for expediency, they are simply called bipartite circulants. This usage is different than our usage of the term bipartite circulant graph. More precisely, a bipartite circulant (as used in this paper) is a circulant graph which is bipartite. In particular, the family of bipartite circulants is a subclass of the generalized bipartite circulants, the family of graphs studied in [18]. The reader should be aware of the two usages when consulting [18].

Our starting point is the following characterization of bipartite graphs due to Heuberger.

Theorem 3.1. [15, Theorem 1] Let $G=C_{n}\left(s_{1}, \ldots, s_{t}\right)$ be a connected circulant. Then $G$ is bipartite if and only if $n$ is even and $s_{1}, \ldots, s_{t}$ are odd.

Note that partitioning the vertices of a bipartite circulant into parts based on the parity of their index will provide a bipartition of the vertex set. Two bipartite circulant graphs are given in Figure 6.
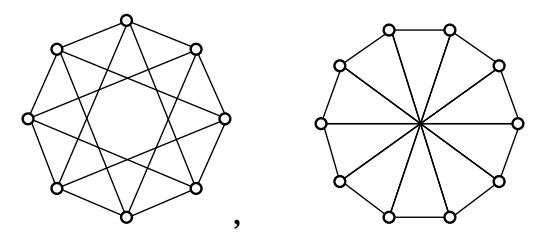

Figure 6: The bipartite circulant graphs $C_{8}(1,3) \cong K_{4,4}$ and $C_{10}(1,5)$

Following Meyers [18], we can represent a bipartite circulant graph using its biadjacency matrix. Recall that if $G$ is a biparitite graph with bipartition $V_{1} \cup V_{2}$ and $m=\left|V_{1}\right|$ and $n=\left|V_{2}\right|$, then we can represent $G$ by the $m \times n$ matrix $A$ where $A_{i j}$ is 1 if there is an edge between vertex $v_{i} \in V_{1}$ and vertex $v_{j} \in V_{2}$, and 0 otherwise. The matrix $A$ is the biadjacency matrix of $G$.

The next lemma describes how to represent the biadjacency matrix of a bipartite circulant graph. Below, $P$ denotes the $n \times n$ permutation matrix corresponding to the $n$ cycle $(123 \cdots n)$. Note that $P^{n}=P^{0}=I_{n}$ and $P^{a}=P^{b}$ if $a \equiv b(\bmod n)$.

Lemma 3.2. Let $G=C_{2 n}\left(s_{1}, \ldots, s_{t}\right)$ be a bipartite circulant graph.

(1) If $s_{t} \neq n$, then the biadjacency matrix of $G$ is

$$
P^{\frac{s_{1}-1}{2}}+P^{\frac{s_{2}-1}{2}}+\cdots+P^{\frac{s_{t}-1}{2}}+P^{n-\frac{s_{t}+1}{2}}+\cdots+P^{n-\frac{s_{2}+1}{2}}+P^{n-\frac{s_{1}+1}{2}} .
$$

(2) If $s_{t}=n$, then the biadjacency matrix of $G$ is

$$
P^{\frac{s_{1}-1}{2}}+P^{\frac{s_{2}-1}{2}}+\cdots+P^{\frac{s_{t}-1}{2}=n-\frac{s_{t}+1}{2}}+\cdots+P^{n-\frac{s_{2}+1}{2}}+P^{n-\frac{s_{1}+1}{2}} .
$$

Proof. This result is implicit in the proof of [18, Theorem 2.2]. In particular, it is shown that if $s \in S$, then $s$ contributes the matrices $P^{\frac{s-1}{2}}$ and $P^{\frac{-s+1}{2}}$ to the biadjacency matrix of $C_{2 n}(S)$ (there is a typo in [18] where the author has an $n$ instead of an $s$ ). Note that $P^{-\frac{s+1}{2}}=P^{n-\frac{s+1}{2}}$. The result now follows by noting that if $s_{t} \neq n$, then each $s_{i} \in S$ gives two distinct matrices, but when $s_{t}=n$, the two matrices $P^{\frac{s_{t}-1}{2}}$ and $P^{-\frac{s_{t}+1}{2}}=P^{n-\frac{s_{t}+1}{2}}$ are the same matrix. 
Remark 3.3. Note that in Lemma 3.2, the exponents of the matrices $P$ satisfy

$$
\frac{s_{1}-1}{2}<\frac{s_{2}-1}{2}<\cdots<\frac{s_{t}-1}{2} \leq n-\frac{s_{t}+1}{2}<n-\frac{s_{t-1}+1}{2}<\cdots<n-\frac{s_{1}+1}{2} \text {. }
$$

We recall two further results from Meyer's paper [18]; we have specialized his results to bipartite circulant graphs of the form $C_{2 n}(S)$.

Lemma 3.4. [18, Theorem 2.4] Suppose that $G=C_{2 n}(S)$ is a connected bipartite circulant graph with biadjacency matrix $P^{i_{1}}+\cdots+P^{i_{r}}$. Then for each unit $a \in \mathbb{Z} / n \mathbb{Z}$ and element $b \in \mathbb{Z} / n \mathbb{Z}$, the graph $G$ is isomorphic to the graph with biadjacency matrix $P^{a i_{1}+b}+\cdots+P^{a i_{r}+b}$ where the exponents are computed modulo $n$.

Theorem 3.5. [18, Corollary 3.5] Suppose that $G=C_{2 n}(S)$ is a connected bipartite circulant graph with biadjacency matrix $P^{0}+P^{1}+P^{2}+\cdots+P^{t}$, with $t \leq n-1$. Then $M(G)=Z(G)=2 t$.

We now come to the main results of this section.

Theorem 3.6. Fix $n, \ell \in \mathbb{N}$ with $\ell \geq 1$ and $n \geq 2 \ell+2$.

(1) If $n$ is odd and $G=C_{2 n}(n-2 \ell, n-2 \ell+2, \ldots, n-2, n)$, then $M(G)=Z(G)=4 \ell$.

(2) If $n$ is even and $G=C_{2 n}(n-2 \ell-1, n-2 \ell+1, \ldots, n-3, n-1)$, then $M(G)=Z(G)=4 \ell+2$.

Proof. (1) Set $k=\frac{n-1}{2}$. Then by Lemma 3.2(2), the biadjacency matrix of $G$ has the form

$$
P^{k-\ell}+P^{k-\ell+1}+\cdots+P^{k}+P^{k+1}+\cdots+P^{k+\ell} .
$$

Let $b=k-\ell$. Then by Lemma 3.4, $G$ is isomorphic to the graph with biadjacency matrix

$$
P^{k-\ell-b=0}+P^{1}+P^{2}+\cdots+P^{k+\ell-b} .
$$

Since $k+\ell-b=2 \ell$, by Theorem 3.5 we get $M(G)=Z(G)=4 \ell$.

The proof of (2) is similar. Let $k=\frac{n-2}{2}$. By Lemma 3.2, the biadjacency matrix of $G$ has the form

$$
P^{k-\ell}+P^{k-\ell+1}+\cdots+P^{k}+P^{k+1}+\cdots+P^{k+\ell+1} .
$$

Using Theorem 3.4, this graph is isomorphic to the graph with biadjacency matrix

$$
P^{0}+P^{1}+\cdots+P^{k+\ell+1-k+\ell} \text {. }
$$

So, Theorem 3.5 gives us the conclusion $M(G)=Z(G)=2(2 \ell+1)=4 \ell+2$.

For our last result, we require the following result about complete bipartite graphs.

Theorem 3.7. Fix $n, \ell \in \mathbb{N}$ with $n>1, \ell \geq 1$, and $n \geq 2 \ell-1$.

1. If $2 \ell-1 \leq n-1$ and $G=C_{2 n}(1,3, \ldots, 2 \ell-1)$, then $M(G)=Z(G)=4 \ell-2$.

2. If $2 \ell-1=n$ and $G=C_{2 n}(1,3, \ldots, 2 \ell-1)$, then $M(G)=Z(G)=4 \ell-4$.

Proof. The proof of both statements are similar to the proof of Theorem 3.6.

(1) By Lemma 3.2, the biadjacency matrix of $G$ has the form

$$
P^{0}+P^{1}+\cdots+P^{\ell-1}+P^{n-\ell}+\cdots+P^{n-1} .
$$

Adding $\ell$ to each exponent, by Lemma 3.4, the graph $G$ is isomorphic to the graph with the biadjacency matrix

$$
P^{0}+P^{1}+\cdots+P^{\ell-1+\ell} \text {. }
$$

Employing Theorem 3.5 gives us $M(G)=Z(G)=2(2 \ell-1)=4 \ell-2$.

(2) If $G=C_{2 n}(1,3, \ldots, n)$, then the biadjacency matrix is $P^{0}+P^{1}+\cdots+P^{n-1}$, and consequently, $M(G)=$ $Z(G)=2 n-2=2(2 \ell-1)-2=4 \ell-4$ by Theorem 3.5. Alternatively, one notes that $G=K_{n, n}$, and so the result follows, for example, by [4, Observation 3]. 

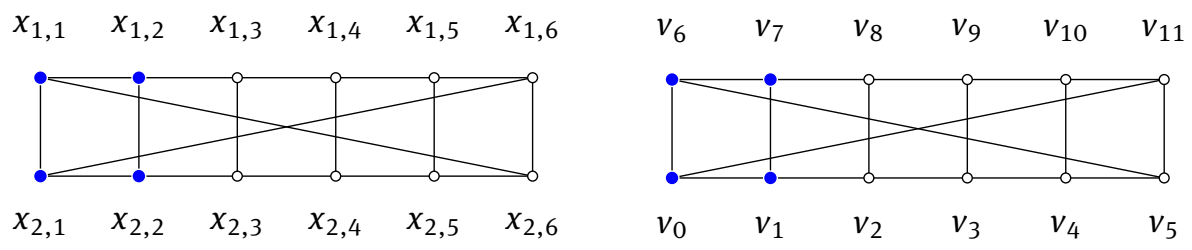

Figure 7: The Möbius ladder $K_{2} \bowtie C_{6} \cong C_{12}(1,6)$ with a zero forcing set.

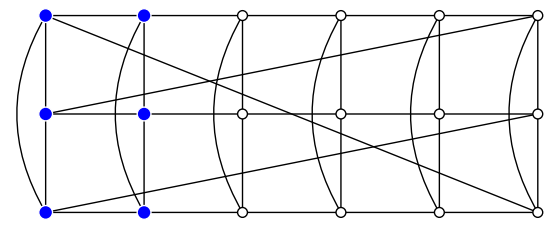

Figure 8: The torus product $K_{3} \bowtie C_{6} \cong C_{18}(1,6)$ with a zero forcing set.

\section{Circulants which are torus products}

In this section we extend the definition of the Möbius ladder to a type of torus product. The zero forcing number for the Möbius ladder was calculated in [2] to be four (e.g., see Lemma 1.2(7)). We compute the zero forcing number for our torus products, and as a corollary, we are able to compute the zero forcing number for a new family of circulant graphs. We also give evidence for a conjecture on the minimum rank of this family.

Recall that the Cartesian product of the graphs $G$ and $H$ with $V(G)=\left\{x_{1}, x_{2}, \ldots, x_{n}\right\}$ and $V(H)=$ $\left\{y_{1}, y_{2}, \ldots, y_{m}\right\}$ is the graph $G \square H$ with vertex set $V=\{(x, y) \mid x \in V(G), y \in V(H)\}$ with two vertices $\left(x_{i}, y_{j}\right),\left(x_{k}, y_{\ell}\right)$ adjacent if either $i=k$ and $y_{j}$ is adjacent to $y_{\ell}$ in $H$, or $j=\ell$ and $x_{i}$ is adjacent to $x_{k}$ in $G$. We position the vertices of $G \square H$ in a $n \times m$ grid such that the $i$-th column contains the vertices $\left(x_{k}, y_{i}\right)$, for $1 \leq k \leq n$, and the $j$-th row contains the vertices $\left(x_{j}, y_{k}\right)$, for $1 \leq k \leq m$. Then $G \square H$ essentially consists of $m$ copies of $G$ as columns and $n$ copies of $H$ as rows. The product $C_{n} \square C_{m}$ can be pictured as a lattice on a torus (see [17]). For $m \geq 3$, define the torus product graph $G \bowtie C_{m}$ to consist of $m$ copies, $G_{1}, \ldots G_{m}$, of $G$ with $G_{i}$ having vertices $x_{1, i}, x_{2, i}, \ldots, x_{n, i}$ with edges between copies as follows: for $1 \leq i \leq m-1$ and $1 \leq k \leq n, x_{k, i}$ is adjacent to $x_{k, i+1}$ and, with subscript addition modulo $n, x_{i, m}$ is adjacent to $x_{i+1,1}$. Then the Möbius ladder is simply the torus product $K_{2} \bowtie C_{m}$ (see for example Figures 7 and 8). Note that the torus product $C_{n} \bowtie C_{m}$ is referred to as a twisted torus in [17].

The following proof takes advantage of the fact that the torus product $K_{n} \bowtie C_{m}$ is locally similar to the Cartesian product $K_{n} \square C_{m}$. Let $G=K_{n} \square C_{m}$ for $m \geq 4$. It was shown in [2] that $Z(G)=M(G)=2 n$. Below we give an alternative argument that $Z(G)=2 n$; the same argument applies to the torus product $K_{n} \bowtie C_{m}$.

Theorem 4.1. Let $G=K_{n} \square C_{m}$ or $G=K_{n} \bowtie C_{m}$. If $m \geq 4$, then $Z(G)=2 n$. If $m=3$ and $n \geq 3$, then $Z(G)=2 n-1$. If $m=3$ and $n=2$, then $M(G)=Z(G)=4$.

Proof. Let $G=K_{n} \square C_{m}$ or $G=K_{n} \bowtie C_{m}$.

First consider the case $m \geq 4$. Assume the vertices are in a grid as described before the theorem. The argument uses the fact that locally, about a column of vertices, the graphs of $K_{n} \square C_{m}$ and $K_{n} \bowtie C_{m}$ both have the subgraph structure $K_{n} \square P_{3}$. (In fact there is an automorphism of $G$ that takes column $G_{i}$ to $G_{k}$ for any $i, k$.)

Observe that if $2 n$ vertices of two adjacent copies of $K_{n}$ are filled, this set is a zero forcing set of $G$. Thus, $|Z(G)| \leq 2 n$.

Let $F$ be a minimum zero forcing set for $G$. Pick a forcing vertex $v \in F$. All but one neighbour of $v$ must be in $F$. Since $G$ is $(n+1)$-regular, $|F| \geq n+1$. Once a forcing is made from $v$, the filled vertices are all those vertices 
in some copy of $K_{n}$ (a column), plus two additional vertices in some copy of $C_{m}$ (a row). These $n+2$ filled vertices are in three consecutive columns, say $L, M$, and $R$, the middle column $M$ being completely filled.

In order for a vertex outside of these three columns to force some other vertex, it must be a filled vertex with at least $n-1$ filled neighbours. If these $n$ vertices were originally in $F$, then $|F| \geq(n+1)+(n-1)=2 n$. Thus, if $|F|<2 n$, then before a vertex outside of the three columns can do any forcing, some vertex in one of the three columns must first force a vertex outside these columns.

Without loss of generality, let $u$ be the first vertex in column $R$ used to force a vertex outside the three columns. Then the remaining vertices in $R$ are already filled. Suppose $r \geq 0$ vertices of column $R$ are in $F$. Then the remaining $n-r$ vertices in column $R$ must have been forced from vertices in column $M$. For any vertex in column $M$ to force a vertex in column $R$, there must already be a vertex in column $L$, in the same row, that is filled. This implies that there must be at least $n-r-1$ vertices in column $L$ that are in $F$ and not adjacent to $v$. Hence $|F| \geq(n+1)+r+(n-r-1)=2 n$. Therefore $Z(G)=2 n$.

Now consider the case $m=3$. Let $F$ be a forcing set. As noted above, for a vertex $v \in F$ to force another vertex, there must be at least $(n-2)$ other vertices of $F$ in the same column as $v$. As labelled above, $M$ must start off with either (a) $n-1$ vertices in $F$ or (b) $n$ vertices in $F$. Note that in case (a), there must be at least one row with vertices of $F$ in both $L$ and $R$. In either case, after a forcing is made, the set of all filled vertices must then contain all the vertices of $M$ and two vertices, one in $L$ and one in $R$, adjacent to a common vertex in $M$. If the zero forcing set $F$ contains $\ell$ vertices of $L$ and $r$ vertices of $R$, then the vertices of $M$ could force at most $\ell$ vertices of $R$ and $r$ vertices of $L$. Thus, after forcing, at most $\ell+r$ vertices of $L$ (and their corresponding vertices in $R$ are filled). If $\ell+r<n-1$, then no further forcing can occur. Thus $\ell+r \geq n-1$. In fact, if $t$ is the number of rows that have a vertex of $F$ in $L$ and a vertex of $F$ in $R$, then $\ell+r-t \geq n-1$. In case (a), $t \geq 1$ and in case (b) $t \geq 0$. It follows that $|F| \geq 2 n-1$. To construct a minimum zero forcing set, let $F$ consist of the first $n-1$ vertices of $L$ (with $L$ as column 1), the first $n-1$ vertices of $M$ (with $M$ as column 2), and the first vertex of $R$ (column 3). Then we claim that $F$ is a forcing set with exactly $2 n-1$ vertices. In particular $x_{2,1}$ can force $x_{n, 1}$ if $G=K_{n} \bowtie C_{m}$ and $x_{1,1}$ can force $x_{n, 1}$ if $G=K_{n} \square C_{m}$. Further $x_{1,2}$ can force $x_{n, 2}$. From here the remaining vertices in $R$ can be forced by vertices in $M$. Thus, $Z(G)=2 n-1$.

Finally, for the case $m=3$ and $n=2, G$ is a Möbius ladder and so by [2], $M(G)=Z(G)=4$.
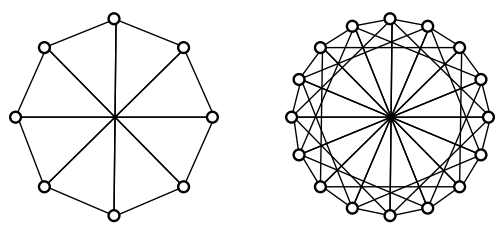

Figure 9: The circulant graphs $C_{8}(1,4) \cong K_{2} \bowtie C_{4}$ and $C_{16}(1,4,8) \cong K_{4} \bowtie C_{4}$.

Remark 4.2. As seen above, the proof takes advantage of the shared local structure of the Cartesian and torus products. Note the only difference between the two graphs is the particular permutation of adjacencies between the first column $G_{1}$ and the last column $G_{n}$. As such, the same result holds true for a much larger class of graphs, if all of the $n$ ! permutations of the adjacencies between the first and last column are considered, not just the two specified by $G \square C_{m}$ and $G \bowtie C_{m}$.

Theorem 4.1 can now be applied to the study of circulant graphs. In the next theorem we assume $m \geq 2$ since if $m=1$ then the graph considered is a consecutive circulant which is already discussed in Theorem 2.5.

Theorem 4.3. Fix $n \geq 3$, and let $G=C_{n m}(1, m, 2 m, \ldots, b m)$ with $b=\left\lfloor\frac{n}{2}\right\rfloor$. If $m=2$, then $Z(G)=n+1$. If $m=3$, then $Z(G)=2 n-1$. If $m \geq 4$, then $Z(G)=2 n$. 
Proof. Let $G=C_{n m}(1, m, 2 m, \ldots, b m)$ with $b=\left\lfloor\frac{n}{2}\right\rfloor$. Then $G$ can be obtained by combining the edges of $C_{n m}(1) \cong C_{n m}$ and $C_{n m}(m, 2 m, \ldots b m) \cong m C_{n}(1,2, \ldots, b)=m K_{n}$. One can then observe that for $m \geq 3$, $C_{n m}(1, m, 2 m, \ldots, b m)$ is the torus product $K_{n} \bowtie C_{m}$ (see for example, Figures 8 and 9). The zero forcing number can then be obtained from Theorem 4.1.

If $m=2$, then $G$ is a $(n+1)$-regular graph and by Lemma 1.2(1), $Z(G) \geq n+1$. Taking any vertex $v$ and all but one of its neighbours provides a zero forcing set of size $n+1$. In particular, $v$ will force its only unfilled neighbour. The remaining $n-2$ unfilled vertices can be forced consecutively from the neighbours of $v$ with subscripts that have the same parity as that of $v$.

We expect that $M(G)=Z(G)$ for all the graphs in Theorem 4.3. Using special matrices, Theorem 4.5 and Theorem 4.7 demonstrate that $M(G)=Z(G)$ for these graphs when $m=4$ and $m=6$.

An $n \times n$ circulant Hankel matrix $H$ is a matrix for which each row is shifted one position to the left from the row above it with a wrap around to the end of the row. In particular, if the first row of $H$ is $\left(a_{1}, a_{2}, \ldots, a_{n}\right)$, then the $k$ th row of $H$ is $\left(a_{k}, a_{k+1}, \ldots, a_{k-1}\right)$. For example,

$$
H=\left[\begin{array}{rrrr}
1 & 2 & 4 & -2 \\
2 & 4 & -2 & 1 \\
4 & -2 & 1 & 2 \\
-2 & 1 & 2 & 4
\end{array}\right]
$$

is a circulant Hankel. Note that the reverse diagonals of a Hankel matrix are constant and consequently the matrix is symmetric. Let

$$
P=\left[\begin{array}{ccccc}
0 & \cdots & \cdots & 0 & 1 \\
1 & \ddots & & & 0 \\
0 & \ddots & \ddots & & \vdots \\
\vdots & \ddots & \ddots & \ddots & 0 \\
0 & \cdots & 0 & 1 & 0
\end{array}\right] .
$$

Note that if $H$ is a circulant Hankel matrix, then $P H=H P^{T}$ and $P H$ is itself a circulant Hankel matrix.

Lemma 4.4. For $n \geq 3$, there exists an orthogonal circulant Hankel matrix $A$ such that both $A$ and $A-P A$ have no zero entries.

Proof. Let $H$ be a circulant Hankel matrix with first row $\mathbf{a}=\left(1,2,2^{2}, \ldots, 2^{n-2}, w\right)$ and $w=-\frac{2}{3}\left(2^{n-2}-1\right)$. We claim that $H$ has orthogonal rows. Since $H$ is circulant, it is enough to show that the first row of $H$ is orthogonal to every other row of $H$. If $\mathbf{b}$ is row $(k+1)$ of $H, 1 \leq k \leq n-1$, then $\mathbf{b}=\left(2^{k}, 2^{k+1}, \ldots, 2^{n-2}, w, 1,2, \ldots, 2^{k-1}\right)$ and

$$
\begin{aligned}
\mathbf{a b}^{T} & =\sum_{i=0}^{n-k-2} 2^{k+2 i}+2^{n-k-1} w+\sum_{i=0}^{k-2} 2^{n-k+2 i}+2^{k-1} w \\
& =2^{k}\left(\frac{4^{n-k-1}-1}{3}\right)+w 2^{k-1}\left[2^{n-2 k}+1\right]+2^{n-k}\left(\frac{4^{k-1}-1}{3}\right) \\
& =\frac{1}{3}\left[2^{2 n-k-2}-2^{k}+2^{n+k-2}-2^{n-k}\right]+w 2^{k-1}\left[2^{n-2 k}+1\right] \\
& =\frac{1}{3}\left[2^{n-k}+2^{k}\right]\left[2^{n-2}-1\right]+w 2^{k-1}\left[2^{n-2 k}+1\right]=0 .
\end{aligned}
$$

Thus $H^{2}=\lambda I$ with $\lambda=\|\mathbf{a}\|^{2}=w^{2}+\sum_{i=0}^{n-2} 2^{2 i}$. Therefore $A=\frac{1}{\sqrt{\lambda}} H$ is an orthogonal circulant Hankel matrix with no zero entries. The fact that $A-P A$ has no zero entries follows from the fact that $H_{i j} \neq H_{i+1, j}$ for $1 \leq i<n$ and $1 \leq j \leq n$.

Theorem 4.5. Given $n \geq 3, M\left(K_{n} \bowtie C_{4}\right)=2 n$. 
Proof. Let $A$ be an $n \times n$ orthogonal circulant Hankel matrix as in Lemma 4.4. Let

$$
K=\left[\begin{array}{cccc}
A & I & O & P^{T} \\
I & B & I & O \\
O & I & -P A & I \\
P & O & I & -P B
\end{array}\right]
$$

with $B=A-P A$. Note that $B$ is a circulant Hankel matrix and $A B=I-P^{T}$ since $P A=A P^{T}$. Also, $B A=I-P$ since $A$ and $B$ are symmetric. Let

$$
E=\left[\begin{array}{cccc}
I & -A & -P^{T} & O \\
O & I & O & O \\
O & O & I & O \\
O & -P & P B & I
\end{array}\right] .
$$

Then, using the fact that $A B=I-P^{T}$ and $B A=I-P$,

$$
E K=\left[\begin{array}{cccc}
O & O & O & O \\
I & B & I & O \\
O & I & -P A & I \\
O & O & O & O
\end{array}\right]
$$

Since $E$ is invertible, it follows that nullity $(K) \geq 2 n$. Note that $K$ is a symmetric matrix with graph $K_{n} \bowtie C_{4}$, since $A, B, P A$ and $P B$ are symmetric matrices with no zero entries. Therefore, $M\left(K_{n} \bowtie C_{4}\right) \geq 2 n$ and thus by Theorem 4.1, $M\left(K_{n} \bowtie C_{4}\right)=2 n$.

Since $K_{n} \bowtie C_{4}$ is the circulant $C_{4 n}(1,4,8, \ldots, 4 b)$ with $b=\left\lfloor\frac{n}{2}\right\rfloor$, we have the following:

Corollary 4.6. If $G=C_{4 n}(1,4,8, \ldots, 4 b)$ with $b=\left\lfloor\frac{n}{2}\right\rfloor$, then $M(G)=Z(G)=2 n$.

Theorem 4.7. Given $n \geq 3, M\left(K_{n} \bowtie C_{6}\right)=2 n$.

Proof. Let $A$ be an $n \times n$ orthogonal circulant Hankel matrix as in Lemma 4.4. Let

$$
K=\left[\begin{array}{cccccc}
A & I & 0 & 0 & 0 & P^{T} \\
I & A & I & 0 & 0 & 0 \\
0 & I & A & I & 0 & 0 \\
0 & 0 & I & P A & I & 0 \\
0 & 0 & 0 & I & P A & I \\
P & 0 & 0 & 0 & I & P A
\end{array}\right] .
$$

The graph of $K$ is $K_{n} \bowtie C_{6}$. If

$$
E=\left[\begin{array}{rrrrrr}
I & -A & 0 & A & -P^{T} & 0 \\
0 & I & 0 & 0 & 0 & 0 \\
0 & 0 & I & 0 & 0 & 0 \\
0 & 0 & 0 & I & 0 & 0 \\
0 & 0 & 0 & 0 & I & 0 \\
0 & -P & P A & 0 & -P A & I
\end{array}\right],
$$

then

$$
E K=\left[\begin{array}{cccccc}
0 & 0 & 0 & 0 & 0 & 0 \\
I & A & I & 0 & 0 & 0 \\
0 & I & A & I & 0 & 0 \\
0 & 0 & I & P A & I & 0 \\
0 & 0 & 0 & I & P A & I \\
0 & 0 & 0 & 0 & 0 & 0
\end{array}\right],
$$

noting that $A P A=A A P^{T}=A A^{T} P^{T}=P^{T}$. Since that first and last $n$ rows of $E K$ are zero, and $E$ is invertible, it follows that $M(G) \geq 2 n$ and by Theorem 4.1, $M(G)=2 n$. 
Corollary 4.8. If $G=C_{6 n}\left(1,6,12, \ldots, 6\left\lfloor\frac{n}{2}\right\rfloor\right)$ then $M(G)=Z(G)=2 n$.

Remark 4.9. Based upon Corollaries 4.6 and 4.8, we wonder if $G=C_{m n}\left(1, m, 2 m, \ldots, m\left\lfloor\frac{n}{2}\right\rfloor\right)$ implies $M(G)=$ $Z(G)$ in general.

There is another circulant graph that is isomorphic to a torus product. In particular, the graph $C_{n m}(1, m) \cong$ $C_{n} \bowtie C_{m}$. (Note that for $m \neq n, C_{m} \bowtie C_{n}$ is not isomorphic to $C_{n} \bowtie C_{m}$.)

The following theorem mimics a result [5] on the zero forcing number of the Cartesian product $C_{n} \square C_{m}$, which is not surprising since locally, the graph is the same as $C_{n} \bowtie C_{m}$. In particular, it was shown in [5] that, with $n \geq m \geq 3$, then $M\left(C_{m} \square C_{m}\right)=Z\left(C_{m} \square C_{m}\right)=2 m-1$ if $m$ is odd, and otherwise $M\left(C_{n} \square C_{m}\right)=Z\left(C_{n} \square C_{m}\right)=$ $2 m$.

We do not know if $M(G)=Z(G)$ in general for $G=C_{n} \bowtie C_{m}$, but the zero forcing number is bounded above in same way as the Cartesian product, except when $m=n$ and $m$ is even. In this case, $Z\left(C_{m} \bowtie C_{m}\right)<Z\left(C_{m} \square C_{m}\right)$. The argument is similar to that in [11, Theorem 2.18].

Theorem 4.10. Suppose $n \geq 3$ and $m \geq 3$. Then $Z\left(C_{n} \bowtie C_{m}\right) \leq \begin{cases}2 \min \{n, m\} & \text { if } m \neq n \\ 2 m-1 & \text { if } m=n \text {. }\end{cases}$

Proof. Let $G=C_{n} \bowtie C_{m}$. First note that if two consecutive columns of vertices of $G$ are in a set $F$, then $F$ is a zero forcing set of $G$. In particular, each of these two columns can force all the vertices on a neighbouring column. If two consecutive rows of vertices of $G$ are in $F$, by symmetry one can assume the first two rows of $G$ are in $F$. In this case, one can force the whole third row, left to right: in particular, $x_{2, j}$ can force $x_{3, j}$ as $j$ ranges from 1 to $m$. (Note that $x_{2, m}$ cannot force $x_{3, m}$ until $x_{3,1}$ has been filled.) Consequently, each of the subsequent rows can also be forced. Therefore, $Z(G) \leq 2 \min \{m, n\}$.

Now suppose $m=n$. Let $k=\left\lceil\frac{m}{2}\right\rceil$. Suppose $F$ consists of the $m$ vertices of column $k$, and $m-1$ vertices of column $k+1$, namely $\left\{x_{2,(k+1)}, \ldots, x_{m,(k+1)}\right\}$. Then column $k$ can force $m-1$ vertices in column $k-1$, in rows 2 through $m$. Now the two columns with $m-1$ vertices can each force $m-3$ vertices in the columns $k+2$ and $k-2$ respectively, namely in rows 3 though $m-1$. This can be repeated, filling two less vertices in each column until the end columns are reached. If $m$ is odd, then the forcing above will result in the two vertices in each of columns 1 and $m$ being filled. In particular, rows $k$ and $k+1$ will be completely filled, and so the resulting set will force the remaining rows to be filled, as noted at the beginning of the proof. If $m$ is even, then column 1 will have the three vertices $x_{k, 1}, x_{(k+1), 1}$, and $x_{(k+2), 1}$ filled but column $m$ will only have vertex $x_{(k+1), m}$ filled. However, $x_{(k+1), 1}$ can force $x_{k, m}$. At this point, rows $k$ and $k+1$ are completely filled, and so the remainder of the vertices can be forced. Therefore, $Z\left(C_{m} \bowtie C_{m}\right) \leq 2 m-1$.

Corollary 4.11. If $n, m \geq 3$, then $Z\left(C_{m^{2}}(1, m)\right) \leq 2 m-1$, and if $n \neq m$, then $Z\left(C_{n m}(1, t)\right) \leq 2 \min \{m, n\}$ for each $t \in\{m, n\}$.

Corollary 4.11 deals with the case that $n \geq 3$. (An earlier version of the $C_{m^{2}}(1, m)$ case is found in [12].) For the case with $n=2$, see Theorem 5.2. Determining $M(G)$ for the graphs $G$ in Corollary 4.11 does not seem to be straightforward. For the case with $m=n=3$, the following theorem demonstrates that $M(G)=Z(G)$.

Theorem 4.12. If $G=C_{9}(1,3)$, then $M(G)=Z(G)=5$.

Proof. Let $G=C_{9}(1,3)$. By Corollary 4.11, $Z(G) \leq 5$. By Theorem 1.1, it is enough to show that $M(G) \geq 5$. Let

$$
A=\left[\begin{array}{rrr|rrr|rrr}
-\frac{1}{8} & \frac{3}{4} & -\frac{1}{2} & 1 & 0 & 0 & 0 & 1 & 0 \\
\frac{3}{4} & -2 & \frac{1}{2} & 0 & 1 & 0 & 0 & 0 & 1 \\
-\frac{1}{2} & \frac{1}{2} & -\frac{3}{4} & 0 & 0 & 1 & 1 & 0 & 0 \\
\hline 1 & 0 & 0 & \frac{48}{5} & -\frac{12}{5} & -\frac{24}{5} & -\frac{16}{5} & 0 & 0 \\
0 & 1 & 0 & -\frac{11}{5} & \frac{6}{5} & \frac{4}{5} & 0 & \frac{12}{5} & 0 \\
0 & 0 & 1 & -\frac{24}{5} & \frac{4}{5} & \frac{4}{5} & 0 & 0 & -\frac{2}{5} \\
\hline 0 & 0 & 1 & -\frac{16}{5} & 0 & 0 & -\frac{2}{5} & -\frac{4}{5} & -\frac{3}{5} \\
1 & 0 & 0 & 0 & \frac{12}{5} & 0 & -\frac{4}{5} & \frac{24}{5} & \frac{6}{5} \\
0 & 1 & 0 & 0 & 0 & -\frac{2}{5} & -\frac{3}{5} & \frac{6}{5} & -\frac{3}{10}
\end{array}\right] .
$$


Then, $A \in \mathcal{S}(G)$ and $\operatorname{rank}(A)=4$ and, therefore, $M(G) \geq 5$.

For the family of circulant graphs of Corollary 4.11, we can also find a lower bound using the following result based on the girth of a graph. The girth of a graph $G$ is size of the smallest cycle in $G$.

Theorem 4.13. [7] Let $G$ be a graph with girth $g \geq 3$ and minimum degree $\delta \geq 2$. Then

$$
Z(G) \geq(g-3)(\delta-2)+\delta .
$$

The above theorem was first conjectured in [8], and proved in some special cases. The proof of Theorem 4.13 was completed in [7] (and see the references there). Theorem 4.13 is now applied to $C_{m n}(1, t)$.

Theorem 4.14. Let $t \in\{n, m\}$ with $n, m \geq 3$. If $G=C_{n m}(1, t)$, then $Z(G) \geq \begin{cases}4 & \text { if } 3 t=n m \\ 6 & \text { otherwise. }\end{cases}$

Proof. Let $G=C_{n m}(1, t)$. The graph $G$ contains a four cycle: $\left\{v_{0}, v_{1}, v_{t+1}, v_{t}\right\}$. Since, $t \geq 3$, in order for $G$ to have a cycle of length three, then $3 t=n m$. In particular, $G$ would contain the 3 -cycle $\left\{v_{0}, v_{t}, v_{2 t}\right\}$. The theorem then follows from Theorem 4.13 since $G$ is 4-regular.

In the case $n=3$ the zero forcing number equals the lower bound given in Theorem 4.14.

Theorem 4.15. If $G=C_{3 m}(1,3)$, then $Z(G)= \begin{cases}6 & \text { if } m>3 \\ 5 & \text { if } m=3\end{cases}$

Proof. When $m=3$, the result follows from Theorem 4.12. When $m>3$, the upper bound of Corollary 4.11 and the lower bound of Theorem 4.14 agree.

In this section we considered graphs $C_{n m}(1, t)$ with $t \in\{n, m\}$ and $n, m \geq 3$. In the next section we consider the case with $m=2$.

\section{The cubic circulant graphs}

In this section, we determine the maximum nullity and zero forcing number for all cubic circulant graphs. A circulant graph is cubic if it is three regular. If $G$ is a cubic circulant graph, then $G=C_{2 m}(a, m)$ for some $a$ with $1 \leq a<m$. The cubic circulant graphs were characterized in [9].

Theorem 5.1. [9] Let $G=C_{2 m}(a, m)$ with $1 \leq a<m$, and let $t=\operatorname{gcd}(a, 2 m)$.

1. If $\frac{2 m}{t}$ is even, then $G \cong t C_{\frac{2 m}{t}}\left(1, \frac{m}{t}\right)$.

2. If $\frac{2 m}{t}$ is odd, then $G \cong \frac{t}{2} C_{\frac{4 m}{t}}\left(2, \frac{2 m}{t}\right)$.

Theorem 5.1 demonstrates that the zero forcing number of a cubic circulant is going to be a multiple of the zero forcing number of a circulant of the form $C_{2 m}(1, m)$ or $C_{2 m}(2, m)$. The zero forcing number is calculated for these classes in Theorems 5.2 and 5.3 respectively.

Theorem 5.2. Suppose $m \geq 2$, and $G=C_{2 m}(1, m)$. Then $M(G)=Z(G)= \begin{cases}3 & \text { if } m=2 \\ 4 & \text { if } m \geq 3\end{cases}$

Proof. Let $G=C_{2 m}(1, m)$. If $m=2$, then $G=K_{4}$ and, hence, $M(G)=Z(G)=3$ by Lemma 1.2(2). Suppose $m \geq 3$. Then $G$ is isomorphic to the Möbius ladder $K_{2} \bowtie C_{m}$, and hence $M(G)=Z(G)=4$ as noted in [2].

Theorem 5.3. Suppose $m \geq 3$ and $G=C_{2 m}(2, m)$. 
1. If $m$ is odd, then $M(G)=Z(G)=\min \{m, 4\}$.

2. If $m$ is even, then $M(G)=Z(G)= \begin{cases}6 & \text { if } m=4 \\ 8 & \text { if } m \geq 6 .\end{cases}$

Proof. Let $G=C_{2 m}(2, m)$. Suppose $m=2 k$ for some positive integer $k$. Then $\operatorname{gcd}(2 m, 2, m)=2$, and so $G \cong 2 C_{2 k}(1, k)$. Thus, $M(G)=Z(G)$ by Theorem 5.2.

Suppose $m$ is odd. Note that $C_{2 m}(2)$ is a subgraph of $G$ consisting of two disjoint cycles of length $m$. Observe that the cycle containing vertex $v_{0}$ consists of the vertices with even subscripts. The other cycle will consist of the vertices with odd subscripts. For any vertex $v_{i} \in V(G)$, its neighbours will be $\left\{v_{i+m}, v_{i+2}, v_{i-2}\right\}$. Thus, besides the edges of the two aforementioned cycles, the graph $G$ also contains the perfect matching consisting of the $m$ edges $\left\{v_{i}, v_{i+m}\right\}$ with $i \in\{0,2,4, \ldots, 2 m-2\}$, using subscript addition modulo $n$. It follows that $C_{2 m}(2, m) \cong C_{m} \square K_{2}$. The result follows from Lemma 1.2(7).

Theorem 5.4. Let $G=C_{2 m}(a, m)$ with $1 \leq a<m$, and let $t=\operatorname{gcd}(a, 2 m)$.

1. If $\frac{2 m}{t}$ is even, then $M(G)=Z(G)= \begin{cases}3 t & \text { if } m=2 t \\ 4 t & \text { if } m \geq 3 t .\end{cases}$

2. If $\frac{2 m}{t}$ is odd, then $M(G)=Z(G)= \begin{cases}m & \text { if } 2 m=3 t \\ 2 t & \text { otherwise. }\end{cases}$

Proof. The theorem follows from Theorem 5.1 and Theorems 5.2 and 5.3, along with Lemma 1.2(5).

Note that when $a$ and $m$ are odd, then the cubic circulant graphs $C_{2 m}(a, m)$ in Theorem 5.4 are further examples of bipartite circulants discussed in Section 3.

\section{Concluding comment}

For every circulant graph $G$ for which we have calculated $M(G)$ and $Z(G)$, these two numbers have been equal. Equality also holds for the extreme cases; when $G=K_{n}$ or $G=C_{n}$. We wonder if equality holds for every circulant graph in general.

Acknowledgments: We thank the referees for their careful reading of the manuscript. To draw the circulant graphs in this paper, we used the $\mathrm{HT}_{\mathrm{E}} \mathrm{X}$ script of Eastman [13]. Some of the results in this paper first appeared in the MSc thesis of Riddell [20]. This research was supported in part by grants held by the last two authors, NSERC RGPIN-2016-03867 and NSERC RGPIN-2019-05412, respectively.

\section{References}

[1] A. Aazami, Hardness results and approximation algorithms for some problems on graphs, $\mathrm{PhD}$ thesis, University of Waterloo, 2008. http://hdl. handle.net/10012/4147.

[2] AIM minimum rank - special graphs work group, Zero forcing sets and the minimum rank of graphs. Linear Algebra Appl. 428 (2008), 1628-1648.

[3] J.S. Alameda, E. Curl, A. Grez, L. Hogben, A. Schulte, D. Young, and M. Young, Families of graphs with maximum nullity equal to zero forcing number. Spec. Matrices 6:1 (2018), 56-67.

[4] W. Barrett, H. Van Der Holst, and R. Loewy, Graphs whose minimal rank is two. Electron. J. Linear Algebra 11 (2004), $258-280$.

[5] K.F. Benson, D. Ferrero, M. Flagg, V. Furst, L. Hogben, V. Vasilevska, and B. Wissman, Zero forcing and power domination for graph products. Australas. J. Combin. 70(2) (2018), 221-235.

[6] F. Boesch, R. Tindell, Circulants and their connectivities. J. Graph Theory 8 (1984), 487-499.

[7] R. Davila, T. Kalinowski, S. Stephen, A lower bound on the zero forcing number. Discrete Appl. Math. 250 (2018), 363-367.

[8] R. Davila, F. Kenter, Bounds for the zero forcing number of graphs with large girth. Theory Appl. Graphs 2 (2015), Art. 1, 8 pp. 
[9] G. Davis, G. Domke, 3-Circulant graphs. J. Combin. Math. Combin. Comput. 40 (2002), 133-142.

[10] L. Deaett, S. Meyer, The minimum rank problem for circulants. Linear Algebra Appl. 491 (2016), 386-418.

[11] L. DeAlba, J. Grout, L. Hogben, R. Mikkelson, and K. Rasmussen, Universally optimal matrices and field independence of the minimum rank of a graph. Electron. J. Linear Algebra 18 (2009) 403-419.

[12] L. Duong, B.K. Kroschel, Zero Forcing Number (2010). Preprint.

[13] B. Eastman, A $\mathrm{BT}_{\mathrm{E} X} \mathrm{X}$ program for creating circulant graphs, GitHub Repository. Available online https://github.com/brydon-zz/circulant, 2014.

[14] S. Fallat, L. Hogben, Minimum rank, maximum nullity, and zero forcing number of graphs. In Handbook of Linear Algebra, 2nd edition, L. Hogben editor, CRC Press, Boca Raton, 2014.

[15] C. Heuberger, On planarity and colorability of circulant graphs. Discrete Math. 268 (2003), 153-169.

[16] L. Hogben, W. Barrett, J. Grout, H. van der Holst, K. Rasmussen, A. Smith, and D. Young. AIM minimum rank graph catalog, 2016. http://admin.aimath.org/resources/graph-invariants/minimumrankoffamilies/\#/cuig.

[17] A. Márquez, A. de Mier, M. Noy, and M.P. Revuelta, Locally grid graphs: classification and Tutte uniqueness. Discrete Math. 266 (2003), 327-352.

[18] S. Meyer, Zero forcing sets and bipartite circulants. Linear Algebra Appl. 436 (2012), 888-900.

[19] M. Muzychuk, Ádám's conjecture is true in the square-free case. J. Combin. Theory Ser. A 72 (1995), 118-134.

[20] M. Riddell, The zero forcing number of circulant graphs. MSc Project, McMaster University (2017).

[21] D. West, Introduction to graph theory. Prentice Hall, Inc., Upper Saddle River, NJ, 1996. 